\title{
Cortical Excitatory Neurons and Glia, But Not GABAergic Neurons, Are Produced in the Emx1-Expressing Lineage
}

\author{
Jessica A. Gorski, ${ }^{1}$ Tiffany Talley, ${ }^{1}$ Mengsheng Qiu, ${ }^{3}$ Luis Puelles, ${ }^{4}$ John L. R. Rubenstein, ${ }^{2}$ and \\ Kevin R. Jones 1 \\ ${ }^{1}$ Department of Molecular, Cellular, and Developmental Biology, University of Colorado, Boulder, Colorado 80309, \\ ${ }^{2}$ Department of Psychiatry, University of California at San Francisco, San Francisco, California 94143-0984, ${ }^{3}$ Department \\ of Anatomical Sciences and Neurobiology, University of Louisville, Louisville, Kentucky 40241, and ${ }^{4}$ Department of \\ Morphological Sciences, Medical School, University of Murcia, E30100 Murcia, Spain
}

By homologous recombination of an internal ribosome entry site and Cre recombinase coding region into the $3^{\prime}$ untranslated region of the mouse Emx1 gene, we have generated a strain of mice, Emx1 IREScre, that expresses the Cre recombinase in a spatial and temporal pattern like that observed for Emx1. When mated to reporter strains, these mice are a sensitive means to fate-map the Emx1-expressing cells of the developing forebrain. Our results demonstrate that radial glia, Cajal-Retzius cells, glutamatergic neurons, astrocytes, and oligodendrocytes of most pallial structures originate from an Emx1-expressing lineage. On the other hand, most of the pallial GABAergic neurons arise outside the Emx1-expressing lineage. Structures that are located near the basal ganglia (e.g., the amygdala and endopiriform nuclei) are not uniformly derived from Emx1-expressing cells.

Key words: Emx1; cell fate; cortex; hippocampus; Cre; GABAergic interneuron; glia
Recent studies suggest that the vertebrate cerebral cortex primordium (pallium) is subdivided into at least four primary progenitor zones, medial, dorsal, lateral, and ventral, giving rise to the hippocampus, neocortex, piriform cortex, and parts of the endopiriform nucleus and amygdala, respectively (Puelles et al., 2000). Furthermore, there is now evidence that these regions integrate glutamatergic projection neurons and astroglia produced by radial glial cells present in pallial progenitor zones and GABAergic local circuit neurons produced in subpallial progenitor zones (for review, see Marin and Rubenstein, 2001). Some cortical oligodendrocytes also appear to be produced in the cortex, although others may be derived from a subcortical source (Spassky et al., 1998). Additional fate mapping and lineage analysis will be useful in testing these ideas.

Site-specific DNA recombinases are a useful tool for lineage analysis (Yamauchi et al., 1999; Chai et al., 2000). In this strategy, a transgenic mouse strain expressing a site-specific DNA recombinase (e.g., Cre) in a subset of cells is bred with a transgenic "reporter" strain in which the reporter gene is only expressed after deletion of DNA sequences by the recombinase. Thus, offspring having both the recombinase and reporter transgenes express the reporter in cells whose ancestors expressed the re-

\footnotetext{
Received Jan. 29, 2002; revised April 8, 2002; accepted April 15, 2002.

This work was supported by a Burroughs-Wellcome New Investigator in Pharmacology Award and grants from the American Cancer Society and the Colorado Council for Research and Creative Work (K.R.J.), National Institutes of Health (J.L.R.R.), and the Spanish Dirección General de Enseñanza Superior and Séneca Foundation (L.P.). We thank Susan Tamowski and Ryan Hebert for technical assistance, Gino Corte for anti-Emx1 antibody, Andras Nagy and Susan Dymecki for mouse strains, and Min Han for fluorescence microscope access.

Correspondence should be addressed to Kevin R. Jones, 347 UCB, Department of Molecular, Cellular, and Developmental Biology, University of Colorado, Boulder, CO 80309. E-mail: krjones@stripe.colorado.edu.

Copyright (C) 2002 Society for Neuroscience $\quad 0270-6474 / 02 / 226309-\bullet \$ 15.00 / 0$
}

combinase at a previous developmental stage, even if recombinase expression was transient.

To study lineage relationships in the cerebral cortex of mice, we used the Emx1 gene to drive expression of the Cre recombinase. Emx1 encodes a homeodomain protein whose expression is restricted primarily to cortical subdivisions of the telencephalon (Simeone et al., 1992a,b; Briata et al., 1996; Gulisano et al., 1996; Puelles et al., 2000). Emx1 is expressed in both progenitor cells and postmitotic neurons of the medial, dorsal, and lateral pallia; expression is not detectable in the ventral pallium during early stages of development. Neuronal expression in the cortex is restricted to projection neurons (Chan et al., 2001). Mating this Emx1 ${ }^{\text {IREScre }}$ strain to Cre-dependent reporter strains defines lineage relationships between cortical neurons and glia and sheds light on the origins of the cellular constituents of several major telencephalic structures.

\section{MATERIALS AND METHODS}

Generation of targeting construct. An Emx1 cDNA fragment (Qiu et al., 1996) was used to isolate a genomic clone containing the last Emx1 exon. An improved internal ribosomal entry site IREScre gene (Gorski and Jones, 1999) followed by an FRT site-flanked PGKneo selectable marker was inserted into an XhoI site located $120 \mathrm{bp} \mathrm{3'}$ ' of the Emx1 stop codon. The FRT-PGKneo-FRT cassette was generated by annealing oligonucleotides containing FRT sites (FRTNEO linker: 5'-CTAGAATTCGAAGTTCCTATTCCGAAGTTCCTATTCTCTAGAAAGTATAGGAACTTC-3' and 5'-AATTGAAGTTCCTATACTTTCTAGAGAATAGGAACTTCGGAATAGGA ACTTCGAATT-3'; FRTNEOB linker: 5'-AGCTCGAAGTTCCTATTCCGAAGTTCCTATTCTCTAGAAAGTATAGGAACTTCAAGCTTGGCCGCGGAGCTCGGGCC- ${ }^{\prime}{ }^{\prime}$ and $5^{\prime}$ CGAGCTCCGCGGCCAAGCTTGAAGTTCCTATACTTTCTAGAGAATAGGAACTTCGGAATAGGAACTTCG-3') followed by ligation to the EcoRI-HindIII PGKneo fragment from pGEM7(KJ1) (Rudnicki et al., 1992).

Isolation of mouse strain and genetics. All animal procedures were conducted in accord with U.S. Public Health Service guidelines and with 
the approval of the University of Colorado Institutional Animal Care and Use Committee. Targeted embryonic stem cell clones were identified and injected into blastocysts, and mice carrying the mutation were derived using standard methods. Four strains were isolated from independent stem cell clones; all displayed indistinguishable patterns of recombination when mated to reporter strains. One of the four strains was mated to the FLP-4917 Flp recombinase-expressing transgenic strain (Dymecki, 1996) to eliminate the PGKneo selectable marker. The resulting Emx1 ${ }^{\text {IREScre }}$ mice were backcrossed four to eight generations to C57BL/6J and bred to the reporter strains, and offspring were genotyped using PCR.

Western blotting. Emx1 protein was quantified from the neocortex of perinatal $(\mathrm{P} 0)$ wild-type $(n=4)$ and Emx $1^{\text {IREScre }}$ homozygous $(n=3)$ pups. Protein $(15 \mu \mathrm{g})$ was transferred and incubated with an anti-Emx1 antibody and normalized to anti-NeuN (Molecular Probes, Eugene, OR) using the Odyssey detection reagents and software (Li-Cor Biosciences, Lincoln, NE).

Histochemistry and immunohistochemistry. Procedures for 5-bromo-4chloro-3-indoyl- $\beta$-D-galactopyranoside (X-gal) staining and immunocytochemistry were as described previously (Vigers et al., 2000). Primary antibody sources were as follows: anti- $\beta-$ Gal (a gift from C. Yee and T. Finger, University of Colorado Health Sciences Center), anti- $\beta$-Gal (ICN Pharmaceuticals, Auroria, $\mathrm{OH}$ ), anti-calretinin, anti-calbindin, and anti-ChAT (Chemicon, Temecula, CA), anti-GABA, anti-neurofilament, and anti-parvalbumin (Sigma, St Louis, MO), anti-NeuN (Molecular Probes), anti-Rip and anti-RC2 (Developmental Studies Hybridoma Bank), and anti-S100 (Dako, Glostrup, Denmark). Alexa 488 goat antimouse IgG antibody and Alexa 546 goat anti-rabbit IgG antibody (Molecular Probes) were used for detection. Sections were coverslipped with Fluoromount (Fisher Scientific, Pittsburgh, PA), and images were captured using an Axioplan deconvolution microscope (Zeiss, Germany) with an attached Hamamatsu C4742-95 digital camera (Hamamatsu, Bridgewater, NJ) running Openlab software (Improvision, Coventry, UK).

\section{RESULTS}

\section{Insertion of an IREScre into the Emx1 3'-UTR}

Homologous recombination was used to insert an IREScre gene into the exon encoding the $3^{\prime}$ untranslated region of the mouse Emx1 gene, thereby generating bicistronic messenger RNAs encoding both the Emx1 and Cre proteins (Fig. $1 A, B$ ). Homozygous Emx1 $1^{\text {IREScre }}$ mice and transheterozygous Emx $1^{\text {IREScre }} / \mathrm{Emx} 1^{\text {null }}$ mice did not exhibit any of the abnormal phenotypes reported for Emx1 ${ }^{\text {null }} /$ Emx1 $^{\text {null }}$ mice (Qiu et al., 1996; Yoshida et al., 1997) $(n=3$; data not shown). There was no apparent reduction of Emx1 protein in the neocortex of Emx $1^{\text {IREScre }} / \mathrm{Em} \times 1^{\text {IREScre }}$ neonates compared with wild type (Fig. $1 C$ ). Thus, by both genetic and biochemical criteria, insertion of IREScre did not interfere with the function of the Emx1 gene.

\section{Emx1 ${ }^{\text {IRES }}{ }^{\text {re }}$-mediated recombination in specific telencephalic domains}

To characterize Cre recombinase expression, we mated Emx1 IREScre mice to the R26R strain (Soriano, 1999). In the R26R strain, Cre-mediated recombination enables expression of lacZ-encoded $\beta$-Gal. X-gal staining of embryonic day (E) 10.5 Emx $1^{\text {IREScre }} ;$ R26R embryos revealed recombination in the dorsal telencephalon, maxillary branch of the first branchial arch, cranial ganglia VII/VIII and IX/X, and limb ectoderm (Fig. 1D,E). These results are consistent with reported sites of Emx1 expression (Simeone et al., 1992a,b).

At E12.5 nearly every cell of the proliferative and postmitotic zones in the medial, dorsal, and lateral pallia had undergone recombination (Fig. $1 F$ ). Dorsomedially, recombination included the fimbria (cortical hem) and part of the choroid plexus (Fig. $1 G)$. In the ventral pallium, scattered periventricular cells expressed $\beta$-Gal along with cells that extended radially into the mantle and seemed to accumulate subpially (Fig. $1 H, I$ ). In the
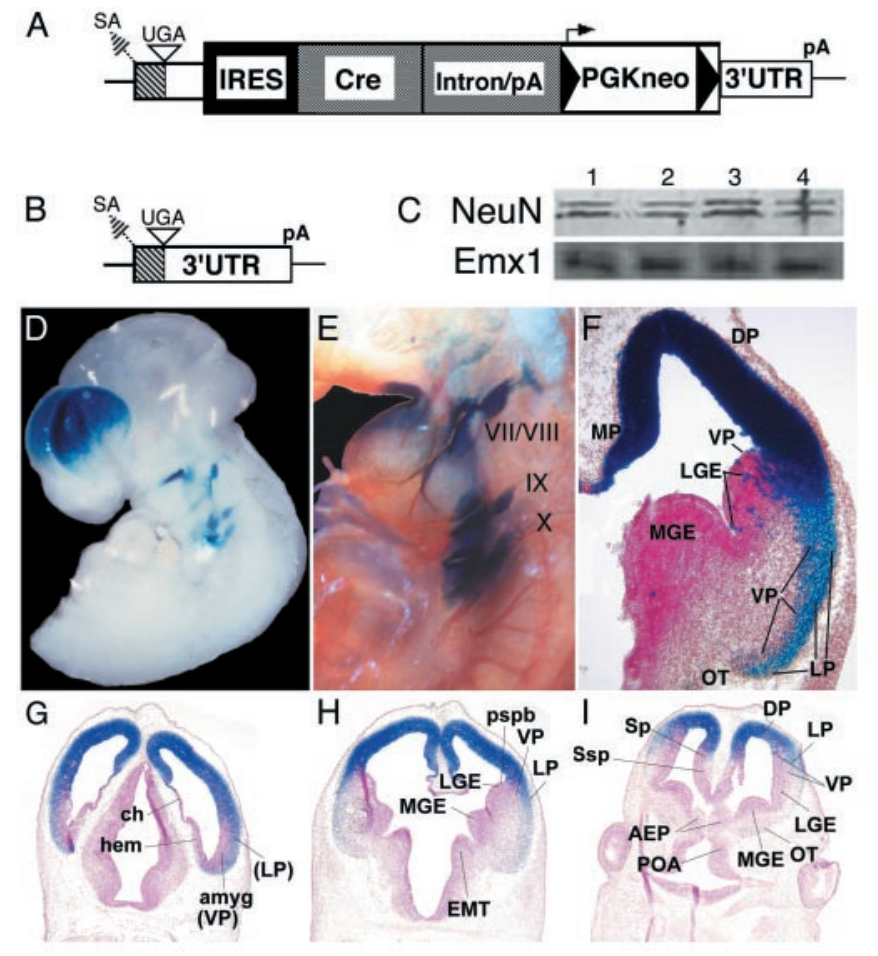

Figure 1. Tissue-specific lox recombination driven by Emx1 ${ }^{\text {IREScre }}$. $A$, Schematic of the Emx1 IREScre targeting construct. $S A$, Splice acceptor; $U G A$, stop codon; $p A$, polyadenylation signal; black triangles, FRT sites; small arrow, PGK promoter. $B$, Schematic of Emx1 exon 3. Hatched box, Coding region; open box, 3'-untranslated. $C$, Western blots of cortical protein from wild-type mice (lanes 1,2$)$ and Emx1 $1^{\text {IREScre }} /$ Emx $^{\text {IREScre }}$ mice (lanes 3, 4), probed with anti-Emx1 or anti-NeuN. D, An X-galstained E10.5 Emx1 ${ }^{\text {IREScre }}$;R26R embryo. E, An E10.5 Emx1 ${ }^{\text {IREScre }}$;R26R embryo X-gal stained and processed for neurofilament immunohistochemistry. Cranial ganglia are numbered. $F-I$, Sections $(10 \mu \mathrm{m})$ of X-gal-stained E12.5 Emx1 ${ }^{\text {IREScre }}$ R26R embryos. AEP, Anterior entopeduncular area; amyg, amygdala; ch, choroid plexus primordium; $D P$, dorsal pallium; $E M T$, eminentia thalami; hem, cortical hem; $M G E$, medial ganglionic eminence; $M P$, medial pallium; $L G E$, lateral ganglionic eminence; $L P$, lateral pallium; $P O A$, preoptic area; $O T$, olfactory tuberculum; $p s p b$, pallial-subpallial boundary; $S p$, pallial septum; $S s p$, subpallial septum; $V P$, ventral pallium. Also see supplemental material at http://www.jneurosci.org.

subpallium, scattered groups of radially oriented recombined cells were apparent in the lateral and medial ganglionic eminences (LGEs, MGEs), with variable numbers in different embryos analyzed (data not shown). Extensive recombination was found in a layer of subpial cells in the mantle of the LGE and MGE. Thus, recombination in E12.5 Emx1 ${ }^{\text {IREScre }}$;R26R embryos was detected not only in all areas of the forebrain reported previously to express Emx1 mRNA, but also in the ventral pallium and in a small subset of LGE and MGE cells.

In the adult, reproducible $(n=4)$ and extensive recombination was seen in the cortical and ependymal layers of the derivatives of the lateral pallium (piriform cortex) (Fig. $2 B-E$ ), dorsal pallium (medial limbic allocortex and neocortex), and medial pallium (hippocampal structures) (Fig. 2E,F). In contrast, only a subset of cells underwent recombination in putative ventral pallial structures (ventral endopiriform nucleus and parts of the amygdala) (Fig. 2B-F).

In the rostral telencephalon, recombination was present in the olfactory bulb, orbital cortex, anterior olfactory nuclei, indusium griseum, and tenia tecta (Fig. $2 A, B$ ). The olfactory bulb had extensive recombination in the mitral cell layer and partial re- 


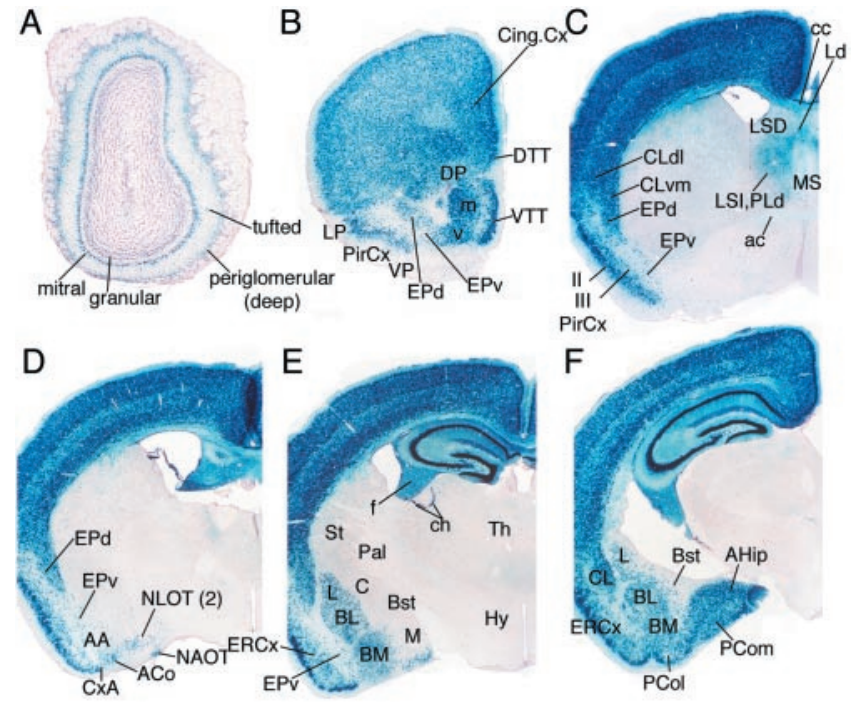

Figure 2. Recombination in the adult Emx $1^{\text {IREScre }}$, R26R brain. $A-F$, Coronal sections $(40 \mu \mathrm{m})$ of X-gal-stained adult Emx1 $1^{\text {IREScre }}$;R26R brain. $A A$, Anterior amygdaloid area; $a c$, anterior commissure; $A C o$, anterior cortical amygdaloid nucleus; AHip, amygdalohippocampal area; $B L$, basolateral amygdala; $B M$, basomedial amygdala; $B s t$, bed nucleus of stria terminalis; $C$, central amygala; $c c$, corpus callosum; $c h$, choroid plexus; Cing. $C x$, cingulate cortex; $C L d l$, dorsolateral claustrum; $C L v m$, ventromedial claustrum; $C x A$, cortex-amygdala transition zone; $D P$, dorsal pallidum; $D T T$, dorsal tenia tectum; $E P d$, dorsal endopiriform nucleus; $E P v$, ventral endopiriform nucleus; $E R C x$, ectorhinal cortex; $f$, fimbria; $H y$, hypothalamus; $L$, lateral amygdala; $L P$, lateral pallidum; $L S D$, lateral septal nucleus dorsal; $L S I$, intermediate lateral septal nucleus; $M$, medial amygdala; $M S$, medial septum; NAOT, bed nucleus of the accessory olfactory tract; $N L O T$ (2), nucleus of lateral olfactory tract, layer II; Pal, pallidum; PCol, posterolateral cortical amygdaloid nucleus; PCom, posteromedial amygdaloid nucleus; $P$ ir $C x$, piriform cortex; $P L d$, paralamboid septal nucleus dorsal; $S t$, striatum; $T h$, thalamus; $V P$, ventral pallidum; $V T T$, ventral tenia tectum. Also see supplemental material at http://www.jneurosci.org.

combination in the granular, internal plexiform, and periglomerular layers. In the septum, the septohippocampal, septofimbrial, and intermediate lateral septal nuclei contained the highest proportion of recombined cells, whereas the main lateral septal nuclei (dorsal and ventral parts) had a lower density, and the medial septal area was negative (Fig. 2C).

In central and caudal regions of the telencephalon, the entire hippocampus, neocortex, and layer II of piriform cortex showed extensive recombination, whereas subcortical structures had only a few recombined cells. The pallial structures that are located between the piriform cortex and the striatum showed a mixed phenotype. Although the dorsolateral claustrum and dorsal endopiriform nucleus showed extensive recombination, the ventral endopiriform nucleus and adjacent deep layer III of the piriform cortex had only scattered X-gal-positive cells (Fig. 2B,C). More caudally, this region contains pallial portions of the amygdala. Recombination was detected in a subset of cells in the lateral, basolateral, and basomedial nuclei of the amygdala (Fig. 2E,F). Superficially, the cortex-amygdala transition zone, the anterior and posterior cortical amygdaloid nuclei, layer II of the nucleus of the lateral olfactory tract, the bed nucleus of the accessory olfactory tract, and the entorhinal cortex contained blue cells. In addition, the posteroventral part of the medial amygdala, together with the associated amygdalo-hippocampal area and amygdalopiriform area, were also X-gal positive (Fig. $2 D$ ). In contrast, very little recombination was detected in a large part of the anterior amygdaloid area and intercalated amygdaloid cell masses (Fig. $2 D)$. No recombination was detected in the ventral endopiriform nucleus (Fig. 2B), the central amygdaloid nucleus (striatal amygdala), the bed nucleus of stria terminalis (pallidal amygdal), and the posterodorsal part of the medial amygdaloid nucleus (Fig. 2E).

Outside of the telencephalon there was little recombination in the brain. The thalamus contained a few scattered recombined cells, possibly from the forebrain/midbrain boundary of the dorsal diencephalon. A few scattered recombined cells in the hypothalamus, habenula, midbrain, and hindbrain were observed (Fig. 2E; and data not shown). Within the midbrain, a subset of cells in the superior colliculus and the periaqueductal gray were recombined (data not shown).

\section{Emx1 and neuronal cell lineages in the telencephalon}

To further identify cells undergoing recombination in Emx $1^{\text {IREScre; }}$ R26R mice, we performed double-label immunofluorescence. In cortex and hippocampus, although many $\mathrm{NeuN}^{+}$cells (a marker for neurons) (Mullen et al., 1992) were also $\beta$-Gal ${ }^{+}(88 \%$ in visual cortex; $n=1567$ cells), $12 \%$ were not. Because of the evidence supporting an extracortical origin for many GABAergic inhibitory interneurons (Marin et al., 2000), we analyzed the relationship between $\beta$-Gal and interneuron markers in Emx1 ${ }^{\text {IREScre }}$;R26R mice. There was little evidence for colocalization of GABA and $\beta$-Gal in the visual cortex (Fig. $3 A-C$ ), where $<2 \%$ of $\mathrm{GABA}^{+}$neurons expressed $\beta$-Gal, and no evidence for recombined parvalbumin and calretinin-positive cells ( $n=2$ mice; 100-135 cells scored per mouse per marker). Afferent fibers may have contributed the faint GABA staining responsible for scoring the few cells as positive. Consistent with these observations in adult mice, we found that calbindin (expressed by precursors of cortical GABAergic neurons) and $\beta$-Gal were not colocalized in E12.5 Emx1 ${ }^{\text {IREScre }}$;R26R cortical plate (Fig. $3 D, F)$. The absence of $\beta$-Gal in $\mathrm{GABA}^{+}$cells was not caused by an inability of the R26R reporter to express in these cells, because $\beta$-Gal staining was readily detected using a different Cre transgene that caused recombination in these cells (data not shown).

As a second approach to determine whether interneurons are generated outside the Emx1 lineage, we analyzed $\beta$-Gal expression in Emx1 $1^{\text {IREScre }}$; $/ A P$ mice, which express $\beta$-Gal in cells that have not undergone Cre-mediated recombination and express alkaline phosphatase in cells that have (Lobe et al., 1999). We found that near-identical proportions of GABA-positive cells were $\beta$-Gal positive in Z/AP and Emx $1^{\text {IREScre }} ; \mathrm{Z} / \mathrm{AP}$ mice (80 and $77 \%$, respectively; $n=145$ cells per genotype). Thus, analysis using both the $\mathrm{Z} / \mathrm{AP}$ and $\mathrm{R} 26 \mathrm{R}$ reporters indicates that all or nearly all GABAergic neurons originate outside the Emx1 lineage.

In adult Emx1 ${ }^{\text {IREScre }} ; \mathrm{R} 26 \mathrm{R}$ striatum, a subpopulation of recombined cells expressed calbindin (Fig. 3G-I), which is used as a marker for medium spiny neurons. On the other hand, recombined striatal cells did not express proteins characteristic of striatal interneurons, including choline acetyltransferase, neuropeptide $\mathrm{Y}$, and parvalbumin (Fig. $3 J-L)(n=2$ mice; $100-250$ cells per mouse per marker).

In neocortical layer 1 of perinatal (P4) Emx $1^{\text {IREScre }} ; \mathrm{R} 26 \mathrm{R}$ pups, we observed near-complete colocalization between $\beta$-Gal and calretinin, likely corresponding to Cajal-Retzius cells (Fig. $4 A-F)$. The few calretinin ${ }^{+} / \beta-\mathrm{Gal}^{-}$cells could be inhibitory interneurons. In E12.5 Emx1 ${ }^{\text {IREScre }} ;$ R26R embryos, radial glia, 

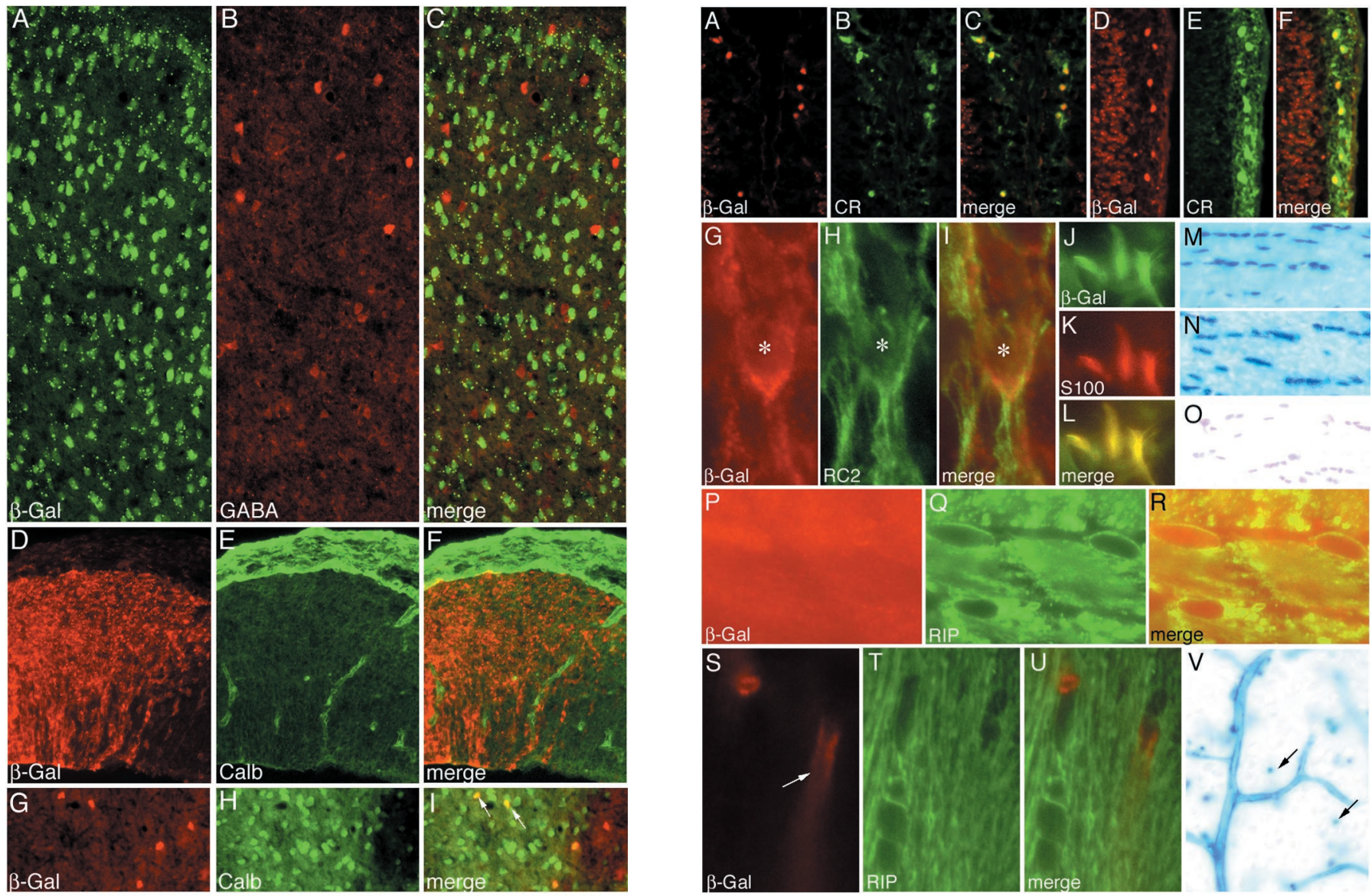

erge

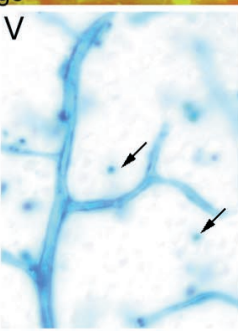

Figure 4. Cajal-Retzius cells, astrocytes, and oligodendrocytes. $A-F$, Retrosplenial cortex $(A-C)$ and visual cortex $(D-F)$ of P4 Emx1 ${ }^{\mathrm{IRES} c r e} ; \mathrm{R} 26 \mathrm{R}$ mice probed with anti-calretinin and anti- $\beta$-Gal. $G-I, \quad$ E12.5 $\mathrm{Emx}^{\mathrm{IRES} c r e} ; \mathrm{R} 26 \mathrm{R}$ cortical plate probed with anti-RC2 and anti- $\beta$-Gal. Asterisk indicates nucleus. $J-L$, Cultured $\mathrm{Em} \times 1^{\mathrm{IRES} c r e} ; \mathrm{R} 26 \mathrm{R}$ astrocytes probed with anti- $\beta$-Gal and anti-S-100. $M-O, \mathrm{X}$-gal-stained adult Emx $1^{\mathrm{IRES} c r e}$; R26R corpus callosum $(M)$, fimbria $(N)$, and anterior commissure $(O)$. $P-U, \mathrm{Em} \times 1^{\mathrm{IRES} c r e} ; \mathrm{R} 26 \mathrm{R}(P-R)$ and $\mathrm{Em} \times 1^{\mathrm{IRES} c r e} ; \mathrm{Z} / \mathrm{AP}(S-U)$ corpus callosum probed with anti- $\beta$-Gal and anti-Rip. $S$, Arrow indicates blood vessel. $V$, Adult Emx1 ${ }^{\text {IREScre }}$; Z/AP neocortex. Blood vessels and scattered cells (arrows) are X-gal stained.

We next sought to determine whether astrocytes are part of the Emx1 lineage. Because S-100-positive astrocytes did not express robust levels of $\beta$-Gal in brain sections prepared from fully Cre-recombined R26R mice (data not shown), we isolated astrocytes from the neocortex of perinatal (P1) Emx1 ${ }^{\text {IREScre }} ; \mathrm{R} 26 \mathrm{R}$ pups and cultured them for $4 \mathrm{~d}$ in vitro. Under these conditions, all S-100-positive cells also expressed $\beta$-Gal, indicating that neocortical astrocytes had undergone recombination (Fig. 4J-L). To corroborate this result, S- $100^{+} / \beta-\mathrm{Gal}^{+}$cells were not detected in the cortex of adult Emx1 $1^{\text {IREScre }}$ Z/AP mice (data not shown).

\section{DISCUSSION}

The Emx $1^{\text {IREScre }}$ mouse is a sensitive tool for determining the fate of Emx1-expressing cells. The specificity of this approach is supported by the fact that the Emx1 $1^{\text {IREScre }}$-induced recombination was primarily restricted to telencephalic regions known to express Emx1 mRNA and protein. Recombination was found in a few telencephalic regions that previously were not reported to express Emx1. These discrepancies could result from the high sensitivity of X-gal staining, transient expression of Emx1 mRNA
$4 V$ ) indicating that endothelial cells had not undergon recombination. 
not previously noted, or migration of transiently Emx1-expressing progenitors. It is also possible that Emx1 is spuriously expressed because of the insertion of the IREScre, but this seems unlikely because mis-expression was not detected elsewhere in the animal.

\section{Emx $1^{\text {IREScre }}$-induced recombination in regions of the pallium}

High levels of recombination were detected in progenitor cells and projection neurons of most regions of the pallium, including the medial, dorsal, and lateral pallium. Lower levels of recombination were present in the putative ventral pallial progenitors and their derivatives. In situ hybridization did not detect clear expression in the ventral pallium in midgestation mice (Puelles et al., 2000). The derivatives of the ventral pallium have been hypothesized to include parts of the claustrum, endopiriform nuclei, and amygdalar complex (Fernandez et al., 1998; Puelles et al., 1999, 2000). Within the amygdala, the basomedial, lateral, anterior, and intercalated nuclei are postulated to be produced in part by the ventral pallium (Puelles et al., 2000). Here we found some support for this model (e.g., the ventral endopiriform nucleus and the anterior and intercalated amygdalar nuclei were mostly unrecombined) (Fig. $2 B-D$ ), but we also found a significant degree of recombination in the area of the claustrum and some recombination in the basomedial and lateral nuclei of the amygdala (Fig. $2 C-F)$.

Two mechanisms may underlie the large number of recombined cells in these regions. First, either induction of Emx1 expression or tangential migration/intercalation of Emx1 ${ }^{+}$progenitors from the adjacent lateral pallium can give rise to recombined clones in the progenitor zone of the ventral pallium. Notably, Fernandez et al. (1998) reported that the ventral pallium was Emx1 ${ }^{-}$at mid-gestation but later became Emx1 ${ }^{+}$in the mouse. Second, tangential migration of recombined cells from more dorsal pallial regions could also contribute recombined cells to the ventral pallium. This type of migration has been observed in guidepost cells of the lateral olfactory tract (Tomioka et al., 2000).

\section{Emx ${ }^{\text {IREScre }}$-induced recombination in regions of the subpallium}

We were surprised to find occasional recombined cells in the subpallium at E12.5 because expression of Emx1 has not been reported previously in this region. The recombined cells have the appearance of radially migrating clones. The mechanisms underlying the origin of these recombined cells can be the same as discussed above for the ventral pallium. The fate of these cells could be the recombined cells found in the adult striatum, pallidum, central and medial amygdala, bed nucleus of the stria terminalis, and septal nuclei. Within the striatum, they do not appear to be interneurons, because no colocalization was detected between $\beta$-Gal and choline acetyl transferase, neuropeptide $\mathrm{Y}$, or parvalbumin.

\section{Identity of Emx ${ }^{\text {IREScre }}$ recombined neurons in the pallium}

In the neocortex and hippocampus, $\sim 88 \%$ of neurons underwent recombination. The vast majority of these must be projection neurons, because $>98 \%$ of GABAergic local circuit neurons did not undergo recombination, indicating that Emx1 is not expressed at any time during development of these cells. A similar finding was reported by Iwasato et al. (2000). This result extends recent studies suggesting that most GABAergic cortical neurons tangentially migrate from the subpallial telencephalon (for review, see Marin and Rubenstein, 2001).

We showed that Cajal-Retzius cells originate within the Emx1 lineage. Previous studies have been contradictory about whether these cells are produced in the pallium or subpallium. Anatomical and marker expression analyses suggested that Cajal Retzius cells originate in the MGE (Lavdas et al., 1999) or have a pallial origin behind the olfactory bulb (Meyer et al., 1998). However, genetic analyses of transcription factors expressed in the pallium and subpallium have suggested a pallial origin (Sussel et al., 1999; Marin and Rubenstein, 2001).

\section{Identity of Emx $1^{\text {IRESCre }}$ recombined glia in the pallium}

We observed recombination in cortical astrocytes and oligodendrocytes. Oligodendrocytes in the corpus callosum and fimbria underwent near-complete recombination, but only scattered oligodendrocytes in the anterior commissure underwent recombination. The anterior commissure forms within the subpallium (Cobos-Sillero et al., 2002), whereas the corpus callosum and fimbria lie in the pallium. Thus, Emx1 ${ }^{+}$pallial progenitors produce oligodendrocytes that specifically interact with axon tracts in the pallium, and Emx1 ${ }^{-}$progenitors produce subpallial oligodendrocytes. Our results are consistent with two models for the origin of oligodendrocytes: that oligodendrocyte precursor cells are restricted to neuroepithelium near the telencephalic-diencephalic junction or that all telencephalic neuroepithelium is able to generate oligodendrocytes (Timsit et al., 1995; Hardy and Friedrich, 1996; Birling and Price, 1998; Spassky et al., 1998). Our data are consistent with the hypothesis that Emx1-expressing progenitors produce projection neurons, oligodendrocytes, and astrocytes, but not inhibitory interneurons, and supports the data indicating that radial glia are precursors for both cortical neurons and astrocytes (for review, see Chanas-Sacre et al., 2000).

\section{REFERENCES}

Birling MC, Price J (1998) A study of the potential of the embryonic rat telencephalon to generate oligodendrocytes. Dev Biol 193:100-113.

Briata P, Di Blas E, Gulisano M, Mallamaci A, Iannone R, Boncinelli E, Corte G (1996) EMX1 homeoprotein is expressed in cell nuclei of the developing cerebral cortex and in the axons of the olfactory sensory neurons. Mech Dev 57:169-180.

Chai Y, Jiang X, Ito Y, Bringas Jr P, Han J, Rowitch DH, Soriano P, McMahon AP, Sucov HM (2000) Fate of the mammalian cranial neural crest during tooth and mandibular morphogenesis. Development 127:1671-1679.

Chan CH, Godinho LN, Thomaidou D, Tan SS, Gulisano M, Parnavelas JG (2001) Emx1 is a marker for pyramidal neurons of the cerebral cortex. Cereb Cortex 11:1191-1198.

Chanas-Sacre G, Rogister B, Moonen G, Leprince P (2000) Radial glia phenotype: origin, regulation, and transdifferentiation. J Neurosci Res 61:357-363

Cobos-Sillero I, Shimamura K, Rubenstein JLR, Martinez S, Puelles L (2002) Fate map of the avian forebrain at stage 8 with quail-chick chimeras. Dev Biol 239:46-67.

Dymecki SM (1996) Flp recombinase promotes site-specific DNA recombination in embryonic stem cells and transgenic mice. Proc Natl Acad Sci USA 93:6191-6196.

Fernandez AS, Pieau C, Reperant J, Boncinelli E, Wassef M (1998) Expression of the Emx-1 and Dlx-1 homeobox genes defines three molecularly distinct domains in the telencephalon of mouse, chick, turtle and frog embryos: implications for the evolution of telencephalic subdivisions in amniotes. Development 125:2099-2111.

Gorski JA, Jones KR (1999) Efficient bicistronic expression of cre in mammalian cells. Nucleic Acids Res 27:2059-2061.

Gulisano M, Broccoli V, Pardini C, Boncinelli E (1996) Emx1 and Emx2 show different patterns of expression during proliferation and differentiation of the developing cerebral cortex in the mouse. Eur J Neurosci 8:1037-1050.

Hardy RJ, Friedrich Jr VL (1996) Oligodendrocyte progenitors are generated throughout the embryonic mouse brain, but differentiate in restricted foci. Development 122:2059-2069.

Iwasato T, Datwani A, Wolf AM, Nishiyama H, Taguchi Y, Tonegawa S, 
Knopfel T, Erzurumlu RS, Itohara S (2000) Cortex-restricted disruption of NMDAR1 impairs neuronal patterns in the barrel cortex. Nature 406:726-731.

Lavdas AA, Grigoriou M, Pachnis V, Parnavelas JG (1999) The medial ganglionic eminence gives rise to a population of early neurons in the developing cerebral cortex. J Neurosci 19:7881-7888.

Lobe CG, Koop KE, Kreppner W, Lomeli H, Gertsenstein M, Nagy A (1999) Z/AP, a double reporter for cre-mediated recombination. Dev Biol 208:281-292.

Marin O, Rubenstein JL (2001) A long, remarkable journey: tangential migration in the telencephalon. Nat Rev Neurosci 2:780-790.

Marin O, Anderson SA, Rubenstein JL (2000) Origin and molecular specification of striatal interneurons. J Neurosci 20:6063-6076.

Meyer G, Soria JM, Martinez-Galan JR, Martin-Clemente B, Fairen A (1998) Different origins and developmental histories of transient neurons in the marginal zone of the fetal and neonatal rat cortex. J Comp Neurol 397:493-518.

Mullen RJ, Buck CR, Smith AM (1992) NeuN, a neuronal specific nuclear protein in vertebrates. Development 116:201-211.

Puelles L, Kuwana E, Puelles E, Rubenstein JL (1999) Comparison of the mammalian and avian telencephalon from the perspective of gene expression data. Eur J Morphol 37:139-150.

Puelles L, Kuwana E, Puelles E, Bulfone A, Shimamura K, Keleher J, Smiga S, Rubenstein JL (2000) Pallial and subpallial derivatives in the embryonic chick and mouse telencephalon, traced by the expression of the genes Dlx-2, Emx-1, Nkx-21, Pax-6, and Tbr-1. J Comp Neurol 424:409-438.

Qiu M, Anderson S, Chen S, Meneses JJ, Hevner R, Kuwana E, Pedersen RA, Rubenstein JL (1996) Mutation of the Emx-1 homeobox gene disrupts the corpus callosum. Dev Biol 178:174-178.

Rudnicki MA, Braun T, Hinuma S, Jaenisch R (1992) Inactivation of MyoD in mice leads to up-regulation of the myogenic HLH gene Myf-5 and results in apparently normal muscle development. Cell 71:383-390.
Simeone A, Acampora D, Gulisano M, Stornaiuolo A, Boncinelli E (1992a) Nested expression domains of four homeobox genes in developing rostral brain. Nature 358:687-690.

Simeone A, Gulisano M, Acampora D, Stornaiuolo A, Rambaldi M, Boncinelli E (1992b) Two vertebrate homeobox genes related to the Drosophila empty spiracles gene are expressed in the embryonic cerebral cortex. EMBO J 11:2541-2550.

Soriano P (1999) Generalized lacZ expression with the ROSA26 Cre reporter strain. Nat Genet 21:70-71.

Spassky N, Goujet-Zalc C, Parmantier E, Olivier C, Martinez S, Ivanova A, Ikenaka K, Macklin W, Cerruti I, Zalc B, Thomas JL (1998) Multiple restricted origin of oligodendrocytes. J Neurosci 18:8331-8343.

Sussel L, Marin O, Kimura S, Rubenstein JL (1999) Loss of Nkx2.1 homeobox gene function results in a ventral to dorsal molecular respecification within the basal telencephalon: evidence for a transformation of the pallidum into the striatum. Development 126:3359-3370.

Timsit S, Martinez S, Allinquant B, Peyron F, Puelles L, Zalc B (1995) Oligodendrocytes originate in a restricted zone of the embryonic ventral neural tube defined by DM-20 mRNA expression. J Neurosci 15:1012-1024.

Tomioka N, Osumi N, Sato Y, Inoue T, Nakamura S, Fujisawa H, Hirata $T$ (2000) Neocortical origin and tangential migration of guidepost neurons in the lateral olfactory tract. J Neurosci 20:5802-5812.

Vigers AJ, Baquet ZC, Jones KR (2000) Expression of neurotrophin-3 in the mouse forebrain: insights from a targeted LacZ reporter. J Comp Neurol 416:398-415.

Yamauchi Y, Abe K, Mantani A, Hitoshi Y, Suzuki M, Osuzu F, Kuratani S, Yamamura K (1999) A novel transgenic technique that allows specific marking of the neural crest cell lineage in mice. Dev Biol 212:191-203.

Yoshida M, Suda Y, Matsuo I, Miyamoto N, Takeda N, Kuratani S, Aizawa S (1997) Emx1 and Emx2 functions in development of dorsal telencephalon. Development 124:101-111. 